\title{
Long-range orientational order in two-dimensional microfluidic dipoles
}

\author{
Itamar Shani ${ }^{1}$, Tsevi Beatus ${ }^{1,2}$, Roy H. Bar-Ziv ${ }^{1 \star}$ and Tsvi Tlusty ${ }^{3 \star}$
}

Dynamic restructuring and ordering are prevalent in driven many-body systems with long-range interactions, such as sedimenting particles ${ }^{1-3}$, dusty plasmas ${ }^{4}$, flocking animals ${ }^{5-7}$ and microfluidic droplets ${ }^{8}$. Yet, understanding such collective dynamics from basic principles is challenging because these systems are not governed by global minimization principles, and because every constituent interacts with many others. Here, we report long-range orientational order of droplet velocities in disordered two-dimensional microfluidic droplet ensembles. Droplet velocities exhibit strong long-range correlation as $1 / \mathbf{r}^{2}$, with a four-fold angular symmetry. The two-droplet correlation can be explained by representing the entire ensemble as a third droplet. The correlation amplitude is non-monotonous with density owing to excluded-volume effects. Our study puts forth a many-body problem with long-range interactions that is solvable from first principles owing to the reduced dimensionality, and introduces new experimental tools to address open problems in many-body non-equilibrium systems ${ }^{9,10}$.

Physical systems with long-range interactions pose a difficult challenge to both theory and experiment and their understanding is considered an open problem, which has attracted much effort in recent years ${ }^{9}$. Long-range hydrodynamic interactions arise in particle-laden fluids, as the motion of particles relative to the surrounding fluid induces a slowly decaying perturbation of the flow field. When the suspension is enclosed in an effective twodimensional (2D) geometry, such as in confined suspensions ${ }^{11,12}$, electrophoresis in capillaries ${ }^{13}$, protein diffusion in membranes ${ }^{14}$, and microfluidic droplets ${ }^{15,16}$, the hydrodynamic perturbations take the form of long-range dipoles. Such dipolar systems, where the exponent of the spatial decay of the interaction equals the dimension, are known to be marginally strong and have therefore attracted special interest ${ }^{9}$. The marginal nature arises from the logarithmic divergence of the interaction with the system size, which leads to intriguing phenomena such as shape dependence, similar to that of dielectrics or magnetic dipoles ${ }^{17}$.

Recently, the collective dynamics of microfluidic droplets has generated a growing interest both as a model system for non-equilibrium dynamics and owing to their practical applications ${ }^{16,18-25}$. Here we generated a highly dynamic and disordered medium with thousands of uniform water-in-oil droplets flowing in a quasi-2D microfluidic channel of width $W=500 \mu \mathrm{m}$ and height $h=10 \mu \mathrm{m}$. The droplets are shaped as discs of uniform radius in the range $R=7-11 \mu \mathrm{m}$ (Fig. 1a). They are in contact with the horizontal floor and ceiling and deform the laminar streamlines of the carrier oil while being dragged at a mean velocity of $u_{\mathrm{d}}=100-200 \mu \mathrm{m} \mathrm{s}^{-1}$ that is roughly four times slower than the oil velocity (Fig. 1b,c). The system is driven far from equilibrium by the imposed flow and operates at a low Reynolds number of $R e \sim 10^{-4}$, where viscosity dominates inertial effects. Each droplet induces a hydrodynamic dipole leading to interactions between the droplets ${ }^{16}$. The dipole is proportional to the velocity difference of oil and droplet, and is aligned with the droplet velocity. Droplet clusters constantly form and break apart erratically, and individual droplets exhibit random, diffusive-like motion due to their interactions with the other droplets ${ }^{20}$. Overall, this highly disordered system exhibits large velocity and density fluctuations that have little to do with thermal energy, consistent with a Peclet number of $P e \sim 10^{7}$.

We tracked the trajectories of the droplets for about $100 \mathrm{~s}$ in a reference frame moving at the mean droplet velocity, and measured the velocity fluctuation of each droplet, $\boldsymbol{\delta} \mathbf{u}(t)=\mathbf{u}(t)-u_{\mathrm{d}} \hat{\mathbf{x}}$, where $\mathbf{u}(t)$ is the droplet's velocity at time $t$ and $\hat{\mathbf{x}}$ is the mean flow direction downstream the channel. The droplets' mean area fraction $\rho_{0}$ was varied in the range of $0.07-0.63$ between measurements and remained roughly constant during each one. The snapshot in Fig. 1d,e shows distinctive patterns of $\boldsymbol{\delta} \mathbf{u}$ for $\rho_{0}=0.18$ with stripes of oriented droplet velocities. These structures demonstrate collective motion with spatial velocity correlations, corresponding to orientational ordering of the hydrodynamic dipoles that are aligned with the velocities. We define the spatial velocity correlation of the measured $\delta u_{x}$ between two points separated by a vector $\mathbf{r}=(r, \theta)$ :

$$
C_{x}(\mathbf{r}) \equiv\left\langle\delta u_{x}\left(\mathbf{r}^{\prime}, t\right) \delta u_{x}\left(\mathbf{r}^{\prime}+\mathbf{r}, t\right)\right\rangle_{r^{\prime}, t}
$$

Here, $\delta u_{x}$ is the $x$ component of $\boldsymbol{\delta} \mathbf{u}$ and averaging is performed over the position $\mathbf{r}^{\prime}$ and time $t$, such that both $\mathbf{r}^{\prime}$ and $\mathbf{r}^{\prime}+\mathbf{r}$ fall within the central half of the channel to avoid boundary effects. A similar definition applies for $\delta u_{y}$. Despite the disordered dynamics, the velocity correlations show remarkable long-range order and symmetry (Fig. 2a-e). Both $C_{x}$ and $C_{y}$ persist over long distances of $r=15-20 R$ before falling below noise level. At large distances, $r=5-20 R$, the correlation exhibits the following hallmarks: decay similar to a power law of $r^{-2}$ (Fig. 2c and Supplementary Fig. 1 for all of the measured densities); anti-symmetry: $C_{y}(r, \theta) \approx-C_{x}(r, \theta)$ (Fig. 2d,e); four-fold angular symmetry, $\sim \cos (4 \theta)$, with positive maxima reflecting a tendency for joint motion in a particular direction, and negative minima that indicate relative motion in opposite directions, that is dilation, contraction or rotation.

To reveal which droplets contribute to the correlations we computed the conditional correlation function defined similarly to equation (1), but limited to positive or negative fluctuations in $x$ and $y$ directions (details in Fig. 2d,e and Supplementary Information). The peaks of the conditional correlations correspond to the characteristic stripy patterns of the orientational order shown in Fig. 1d,e. The correlations manifest the collective dynamics of the droplets: its positive peaks reveal distinct structures and, at the same time, its negative peaks show how these structures are unstable, dynamically breaking and reforming. Consider, for

\footnotetext{
${ }^{1}$ Department of Materials and Interfaces, Weizmann Institute of Science, Rehovot, Weizmann 76100, Israel, ${ }^{2}$ Department of Physics, Cornell University, Ithaca, New York 14853, USA, ${ }^{3}$ Simons Center for Systems Biology, Institute for Advanced Study, Princeton, New Jersey 08540, USA. *e-mail: roy.bar-ziv@weizmann.ac.il; tlusty@ias.edu
} 
a

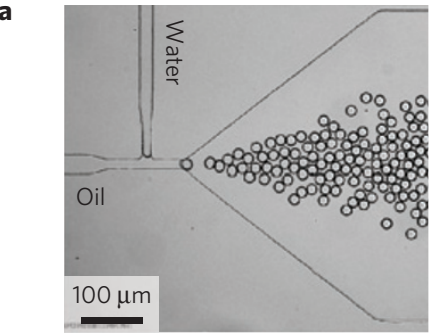

b
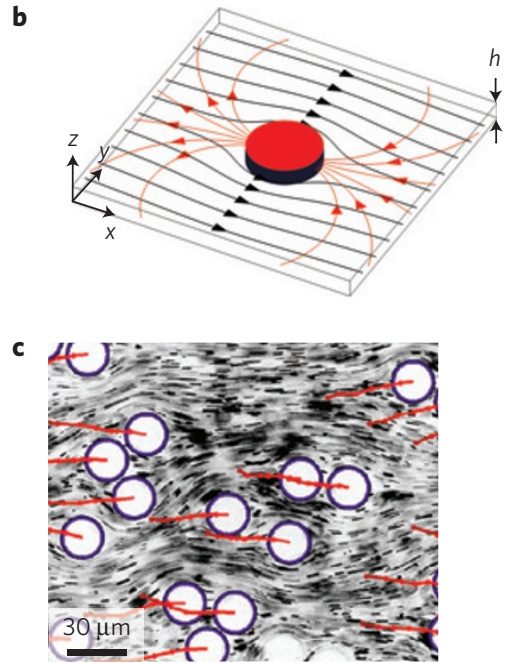

d
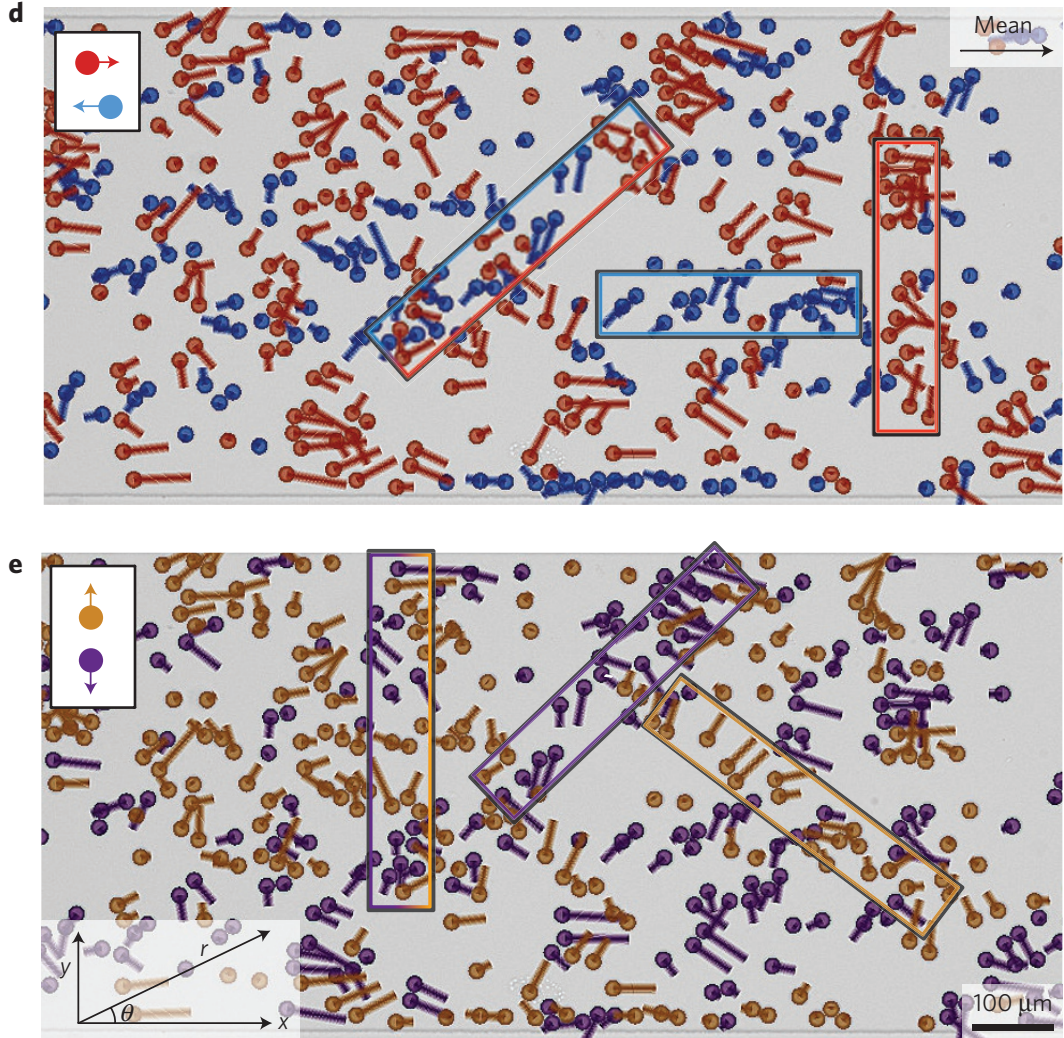

Figure 1 | The microfluidic 2D droplet ensemble and velocity fluctuations. $\mathbf{a}$, The droplets were generated at a T-junction of water and oil streams and injected at constant rate into a channel. b. The droplet geometry is disc-shaped, confined between the floor and the ceiling. The difference between the velocities of the droplet and the surrounding oil (black) induces a dipolar perturbation (red). c, Image of water droplets carried by oil, dispersed with fluorescent beads that moved during camera exposure and highlighted its flow lines (brightness inverted). Red lines show the droplet positions in time. $\mathbf{d}, \mathbf{e}$, Images show ensemble with $\rho_{0}=0.18$. Lines drawn from the centre of each droplet are proportional to $\delta \mathbf{u}$, its velocity relative to the mean (that is, the fluctuation of the dipoles around their mean). d, Red $\delta u_{x}>0$. Blue $\delta u_{x}<0$. e, Yellow $\delta u_{y}>0$. Purple $\delta u_{y}<0$. The rectangular frames highlight the angles along which the colours are typically uniform or mixed, corresponding to positive and negative correlations.

example, a droplet pair with $\theta=90^{\circ}$ : on average, the two droplets move fast together along $x\left(C_{x}^{+}\left(\theta=90^{\circ}\right)>0\right)$ and simultaneously move in opposite directions along $y\left(C_{y}^{ \pm}\left(\theta=90^{\circ}\right)<0\right)$, which either increases or decreases the distance between them. A pair of droplets separated by $\theta \simeq 45^{\circ}$ tend to move together downwards along $y$ and simultaneously move in opposite directions along $x$, which changes $\theta$. Finally, we measured the variance of the velocity, $\left\langle\delta u_{x}^{2}\right\rangle=C_{x}(r=0)$ and $\left\langle\delta u_{y}^{2}\right\rangle=C_{y}(0)$. The variances, in units of $u_{\mathrm{d}}^{2}$, increase with the area fraction $\rho_{0}$, until they peak at $\rho_{0} \sim 0.25$, and then attenuate at higher density (Fig. 2f).

To explain the orientational order manifested by the velocity correlations, we consider the interactions in the $2 \mathrm{D}$ ensemble of hydrodynamic dipoles ${ }^{20}$. In the thin channel, the oil velocity can be decoupled into Poiseuille flow along the $z$ axis perpendicular to the plane, and a 2D potential Stokes flow in the $x y$ plane (Fig. 1b). Far from the confining sidewall boundaries, the disturbance of a single droplet to the oil velocity is the gradient of the $2 \mathrm{D}$ dipole potential, $\Phi(\mathbf{r})=\Delta u \cdot \phi(\mathbf{r})$ with $\phi(\mathbf{r})=R^{2} \cos (\theta) / r$ and $\Delta u=u_{\mathrm{oil}}-u$ is the difference between the unperturbed oil velocity at the droplet position (assuming the droplet is absent) and the droplet velocity. Two opposing forces act on each droplet: a drag by the surrounding oil and a friction force by the solid boundaries at the channel floor and ceiling. As inertia is negligible, the forces are balanced, resulting in a linear relation $u=K u_{\text {oil }}$, where the coupling constant $K \sim 0.25$ depends only on geometry and material properties ${ }^{8,16}$ (Supplementary Information).

Owing to the linearity of Stokes flow, in an ensemble with inter-droplet distances much larger than $R$, the oil velocity is well approximated by a superposition of the droplet dipoles and the uniform oil flow, $\mathbf{u}_{\mathrm{oil}}(\mathbf{r})=\left\langle\mathbf{u}_{\mathrm{oil}}\right\rangle+\sum_{j} \nabla \Phi\left(\mathbf{r}-\mathbf{r}_{j}\right)$. The equation of motion of a droplet at $\mathbf{r}$ is $\mathbf{u}(\mathbf{r})=K \mathbf{u}_{\text {oil }}(\mathbf{r})$, where $\mathbf{u}_{\text {oil }}(\mathbf{r})$ is the velocity of oil including the perturbations of all the other droplets ${ }^{16}$. We represent corrections to this far-field approximation by an effective velocity scale $U$ for the interaction strength that will be determined experimentally (see Supplementary Information):

$$
\mathbf{u}(\mathbf{r})=u_{\mathrm{d}} \hat{\mathbf{x}}+U \sum_{j} \nabla \phi\left(\mathbf{r}-\mathbf{r}_{j}\right)
$$

The correlation between the velocity fluctuations $\delta \mathbf{u}=\mathbf{u}-u_{\mathrm{d}} \hat{\mathbf{x}}$ of two droplets at a distance $\mathbf{r}$ is found from equations (1) and (2) to be the averaged sum over all dipole pairs in the ensemble:

$$
C_{x}(\mathbf{r})=U^{2}\left\langle\sum_{i, j} \partial_{x} \phi\left(\mathbf{r}_{j}-\mathbf{r}^{\prime}\right) \partial_{x} \phi\left(\mathbf{r}_{i}-\mathbf{r}^{\prime}-\mathbf{r}\right)\right\rangle_{\mathbf{r}^{\prime}, t}
$$

and similarly for $C_{y}$. The summation over $i$ and $j$ represents the interaction of the droplet pair with all of the other droplets in the ensemble. Equation (3) can be separated into two parts: a single-droplet contribution $C_{x}^{\mathrm{I}}(\mathbf{r})$, including all terms with $i=j$, that accounts for the velocity change of the two test droplets due to their interactions with a third droplet at $\mathbf{r}_{i}$, and a dual-droplet part, $C_{x}^{\mathrm{II}}(\mathbf{r})$ including all interactions of the pair with two different droplets $i \neq j$.

We first consider the simple case of a randomly positioned ensemble: if the positions of the $i$ th and $j$ th droplets are 


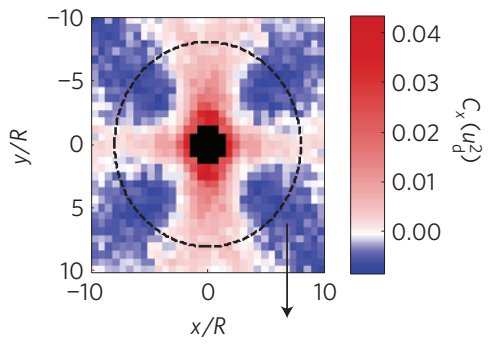

d

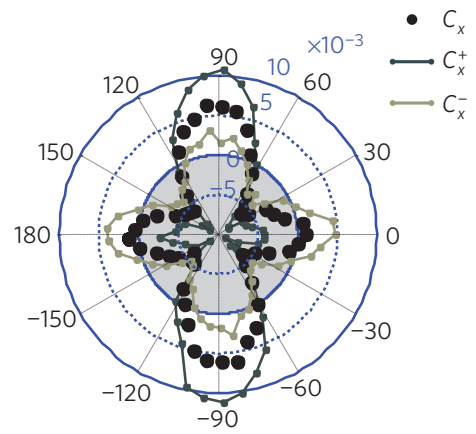

g

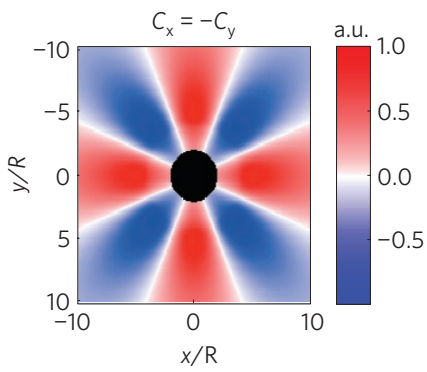

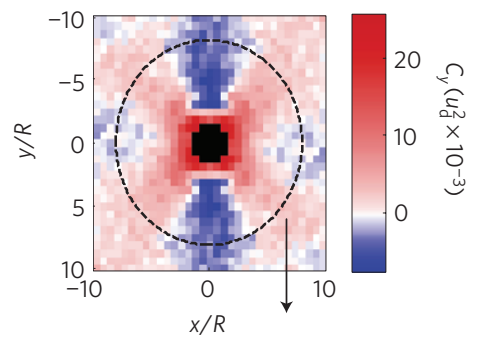

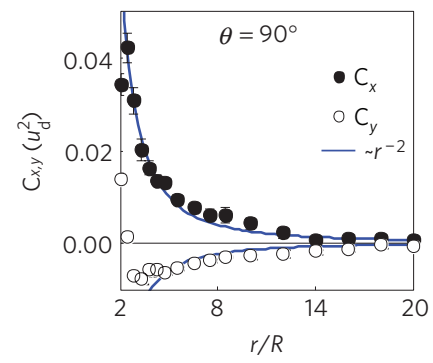

e

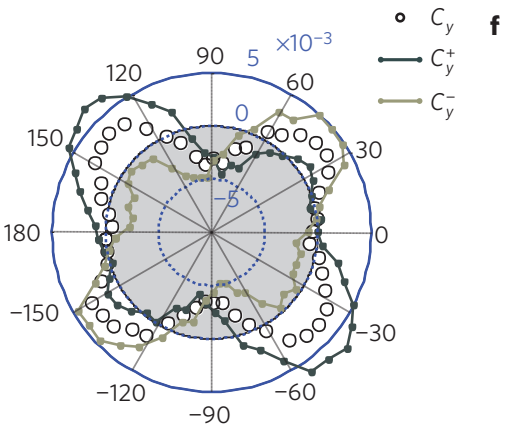

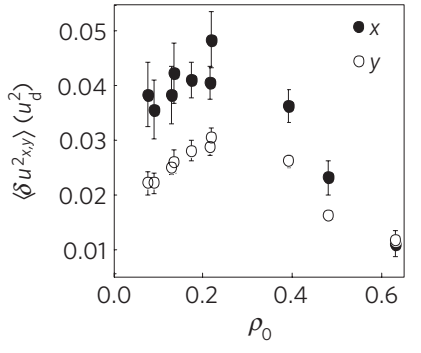

h
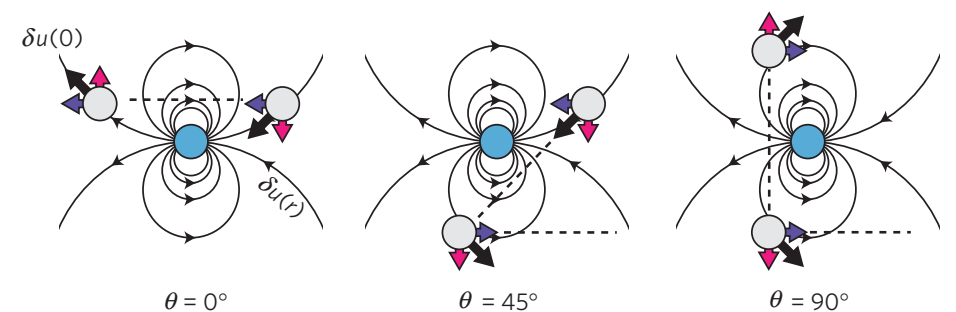

$\theta=90^{\circ}$

Figure 2 | Velocity correlations. a,b, $C_{x}$ and $C_{y}$ versus $x$ and $y$ at $\rho_{0}=0.18 . \mathbf{c}, C_{x}$ and $C_{y}$ versus $r$ for a vertical droplet pair $\left(\theta=90^{\circ}\right)$. The solid line is an $r^{-2}$ curve. d,e, $C_{x, y}$ versus $\theta$ at $r=8 R$. Conditional correlations $C_{x, y}^{ \pm}$reveal structures and dynamics in the droplet ensemble: $C_{x, y}^{+}$includes only the droplets with $\delta u_{x, y}\left(\mathbf{r}^{\prime}, t\right)>0$, and $C_{x, y}^{-}$that is constrained to $\delta u_{x, y}\left(\mathbf{r}^{\prime}, t\right)<0$. The peak at $C_{x}^{+}(\theta=90)$ corresponds to fast droplets $\left(\delta u_{x}>0\right)$, aligned perpendicular to the flow, whereas the peak at $C_{x}^{-}\left(\theta=0^{\circ}\right)$ indicates slow droplets aligned along the flow. The peaks of $C_{y}^{+}$and $C_{y}^{-}$show that droplets aligned along diagonals at $\theta \simeq 45^{\circ}$ have correlated downwards velocity $\left(\delta u_{y}<0\right)$, whereas those aligned along $\theta \simeq-45^{\circ}$ diagonals move upwards $\left(\delta u_{y}>0\right)$. f, Velocity variance $\left\langle\delta u_{x, y}^{2}\right\rangle, \mathbf{g}$, Theoretical $C_{x, y}$ given by equation (4) and Supplementary Eq. 17. h, Velocity fluctuations $\delta u_{x}$ (purple) and $\delta u_{y}$ (violet) of two test droplets typically have parallel or opposite signs owing to their interactions with a third droplet (light blue), depending on $\theta$. Error bars represent uncertainty due to temporal fluctuations of the measured correlations.

independent, then by symmetry the average over the product of their dipolar fields vanishes, $C_{x}^{\mathrm{II}}(\mathbf{r})=0$, and hence $C_{x}=C_{x}^{\mathrm{I}}$. The time average in equation (3) can therefore be replaced by an integral over a uniform density distribution, $n_{0}=\rho_{0} / \pi R^{2}$, that for $r \gtrsim 8 R$ yields (Supplementary Fig. 2 for the full solution):

$$
C_{x}(\mathbf{r})=U^{2} n_{0} \int \mathrm{d}^{2} \mathbf{r}_{i} \partial_{x} \phi\left(\mathbf{r}_{i}\right) \partial_{x} \phi\left(\mathbf{r}_{i}-\mathbf{r}\right)=\rho_{0} U^{2} \cos (4 \theta)(R / r)^{2}
$$

In addition, $C_{y}(\mathbf{r})=-C_{x}(\mathbf{r})$. Equation (4) captures the three salient features of the measured velocity correlations: the $r^{-2}$ power law, the $x y$ anti-symmetry, and the $\cos (4 \theta)$ angular dependence. Interestingly, the velocity correlation is given by the auto-correlation of $\nabla \phi$, which implies that the effect of an entire random ensemble on the test-pair is equivalent to the average effect of a third droplet. To elucidate this effect, Fig. $2 \mathrm{~h}$ shows droplet pairs (grey) at angles $\theta=0^{\circ}, 45^{\circ}, 90^{\circ}$ and a third droplet (light-blue) between each pair. When $\theta=0^{\circ}, 90^{\circ}$ the third droplet's dipole has both a positive contribution to $C_{x}$, because it pushes the pair to the same direction along $x$, and at the same time, a negative contribution to $C_{y}$, because it pushes the pair in opposite directions along $y$. When $\theta=45^{\circ}$, it has a positive contribution to $C_{y}$ and a negative contribution to $C_{x}$.

Remarkably, the velocity correlations originate from the interactions of the pair with the entire ensemble and not from the interaction within the pair. The latter is not included in equation (4) and decays as $(\nabla \phi)^{2} \sim r^{-4}$, much faster than the $\sim r^{-2}$ decay measured at large distances. Moreover, the interaction within the pair cannot explain negative correlations, because the forces that any two droplets apply on each other are identical owing to the symmetry of the dipole field, $\nabla \phi(\mathbf{r})=\nabla \phi(-\mathbf{r})$.

The predicted peaks of the correlations at $\theta= \pm 45^{\circ}$ are observed for $\rho_{0}<0.6$ at an average angle of $\pm 41^{\circ} \pm 2.5^{\circ}$ for $C_{x}$, and $\pm 38^{\circ} \pm 2^{\circ}$ for $C_{y}$ (Fig. 2d,e). In addition, the peaks at $\theta=90^{\circ}$ and $\theta=0^{\circ}$ differ in amplitude, in contrast to the theoretical $\cos (4 \theta)$. These differences may largely be attributed to dependencies between droplet positions due to clustering and density waves ${ }^{20,24}$ that were neglected. To refine equation (4) we consider excluded-volume effects in the low-density limit, $\rho_{0} \ll 1$, and find that the dualdroplet term $C_{x}^{\mathrm{II}}$ no longer vanishes: $C_{x}^{\mathrm{II}}(\mathbf{r})=-4 \rho_{0} C_{x}^{\mathrm{I}}(\mathbf{r})$ for $r \gg R$, whereas $C_{x}^{\mathrm{I}}$ is unchanged, resulting in, $C_{x}(\mathbf{r})=\left(1-4 \rho_{0}\right) C_{x}^{\mathrm{I}}(\mathbf{r})$ (see Supplementary Information). Thus, excluded volume does not alter 


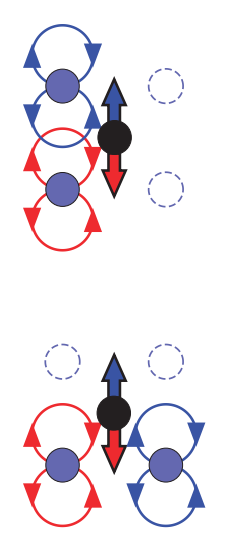

$2 \times$ opposite

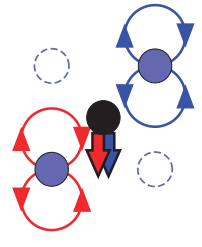

$1 \times$ parallel

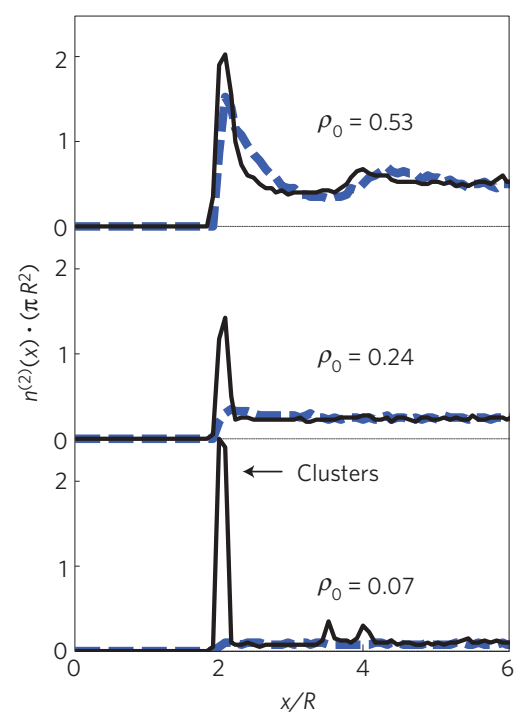

d

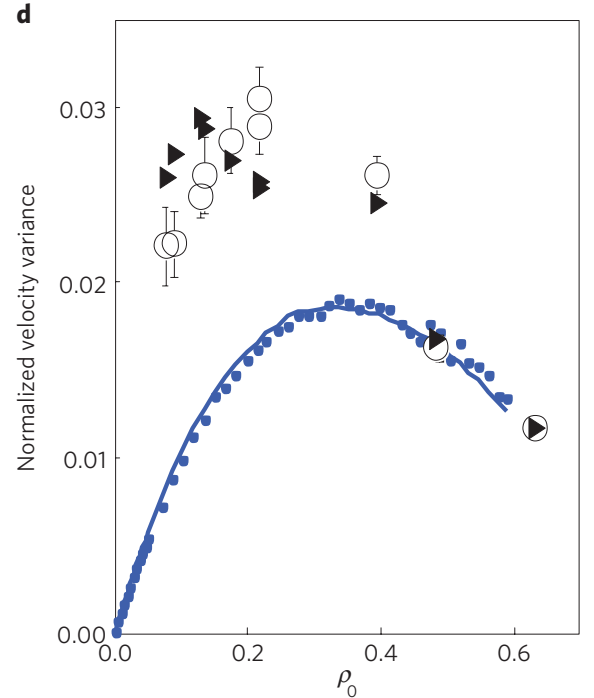

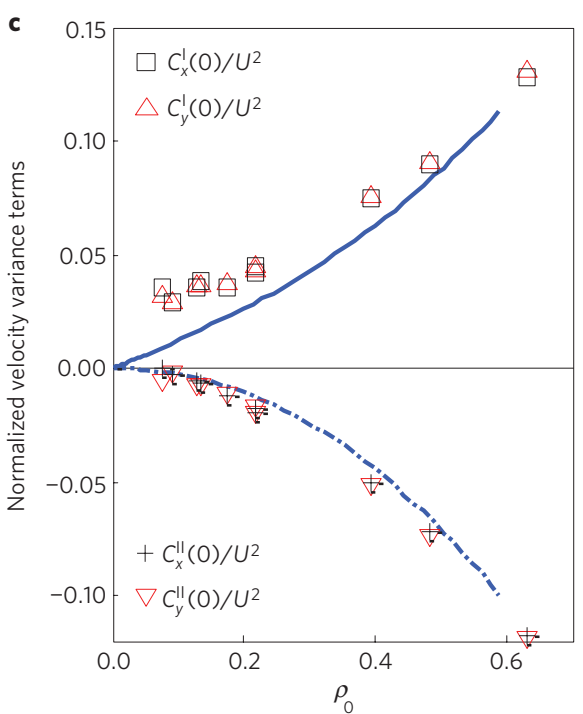

Figure 3 | Velocity variance determined by competition of single- and dual-droplet effects. a, Toy-model: a test droplet (black) surrounded by four available slots (dashed circles) that are occupied by two droplets. As a result of excluded volume, in two out of three configurations, the dipole fields at the test droplet are anti-parallel setting $C_{y}^{\prime \prime}(0)$ negative. $\mathbf{b}$, The pair-distribution function, $n^{(2)}(\mathbf{r})$, the probability to find a droplet $\mathbf{r}$ away from a reference droplet is measured at different $\rho_{0}$, and plotted for $r \| \hat{x}$, peaks at $r \simeq 2 R$, indicating high probability to find droplets in contact owing to clustering. Broken curves are $n^{(2)}(r)$ of randomly placed droplets in silico. c, $C_{x, y}^{1}(0)$ is positive, whereas $C_{x, y}^{\prime \prime}(0)$ is negative. Both increase in magnitude with $\rho_{0}$. Blue curves are $C_{x, y}^{\prime}(0)$ and $C_{x, y}^{\prime \prime}(0)$ calculated in silico. d, The sum $\left[C_{y}^{\prime}(0)+C_{y}^{\prime \prime}(0)\right] / U^{2}$ (right triangle) is similar to $C_{y}(0) / u_{d}^{2}$ (open circle). Blue curves are in silico $C_{y}^{\prime}(0)+C_{y}^{\prime \prime}(0)$ (dots) and $C_{y}(0)$ (solid line), which agree throughout the $\rho_{0}$ range. Error bars represent uncertainty due to temporal fluctuations of the measured correlations.

the spatial structure of the velocity correlations but introduces a density-dependent inhibition to the correlation amplitude, $\left\langle\delta u_{x}^{2}\right\rangle \equiv C_{x}(\mathbf{r}=0)$, which is proportional to $\rho_{0}^{2}$ because it depends on the number of droplet pairs.

The inhibitory effect due to excluded volume reflects a competition between two density-dependent effects that determine the observed peak of the velocity variance $\left\langle\delta u_{x}^{2}\right\rangle=C_{x}^{\mathrm{I}}(\mathbf{r}=0)+C_{x}^{\mathrm{II}}(\mathbf{r}=0)$ (Fig. 2f). Here, $C_{x}^{\mathrm{I}}(0)=U^{2}\left\langle\sum_{i}\left(\partial_{x} \phi\left(\mathbf{r}_{i}-\mathbf{r}^{\prime}\right)\right)^{2}\right\rangle_{\mathbf{r}^{\prime}, t}$, which is positive and $C_{x}^{\mathrm{II}}(0)=U^{2}\left\langle\sum_{i \neq j} \partial_{x} \phi\left(\mathbf{r}_{i}-\mathbf{r}^{\prime}\right) \partial_{x} \phi\left(\mathbf{r}_{j}-\mathbf{r}^{\prime}\right)\right\rangle_{r^{\prime}, t}$. A simple toymodel provides intuition why the second term is always negative owing to excluded-volume effects (Fig. 3a). Consider two droplets that can be positioned at the four corners of a square surrounding a test droplet (black). Then, $C_{x}^{\mathrm{II}}(0)$ is the product of the two fields at the test-droplet's location, averaged over all of the possible configurations. From symmetry it is enough to consider 3 out of the 12 possible configurations, where one droplet is fixed at the bottom left corner and the other can occupy any of the other three spots. In two of these configurations, the dipole fields have opposite directions at the test droplet's position, whereas only in one configuration their directions are aligned. As a result, their average product is negative, hence $C_{x}^{\mathrm{II}}(0)<0$. Without the effect of excluded volume, one would consider a fourth configuration, in which both droplets occupy the same spot, forming a second configuration with aligned fields that nullifies the average product.

To compare the theoretical value of $C_{x}^{\mathrm{I}}(0)+C_{x}^{\mathrm{II}}(0)$ to the measured velocity variance $\left\langle\delta u_{x}^{2}\right\rangle$ (Fig. $2 \mathrm{f}$ ), we measured the spatial pair- and triplet-distribution functions that describe the nonrandom distribution of distances between droplets (Fig. $3 \mathrm{~b}$ and Supplementary Fig. 3). These functions are used as weights in averaging over the ensemble positions in calculating $C_{x}^{\mathrm{I}}(0)$ and 
$C_{x}^{\mathrm{II}}(0)$ (see Supplementary Information). Both functions peak at distances of touching droplets but decay to a constant value for larger distances. In agreement with theory, Fig. $3 \mathrm{c}$ shows that $C_{x}^{\mathrm{I}}(0)$ is positive whereas $C_{x}^{\mathrm{II}}(0)$ is negative, both increasing in magnitude with density $\rho_{0}$, and have similar values for $y$. The computed sum $C_{y}^{\mathrm{I}}(0)+C_{y}^{\mathrm{II}}(0)$ in units of $U^{2}$ fits well to the measured $C_{y}(0)$ (Fig. 3d), which suggests that the interaction velocity scale $U$ is $U \approx u_{\mathrm{d}}$ throughout the measured range of $\rho_{0}\left(C_{x}(0)\right.$ is discussed in the Supplementary Information). The theoretical far-field value, $U=(1-K) u_{\mathrm{d}} \approx u_{\mathrm{d}}$, matches our experimental result.

Finally, we computed the velocity correlations in silico by randomly placing droplets, considering their mutually excluded volume, and evaluating the inter-droplet dipolar forces (see Supplementary Information). In agreement with theory, the numerical calculation shows a $\cos 4 \theta$ symmetry and $r^{-2}$ decay of the correlations (Supplementary Fig. 4), as well as a peak at $\rho_{0}=0.34$ in the velocity variances (Fig. $3 \mathrm{~d}$ ).

Similarly to a nematic liquid crystal, the hydrodynamic dipoles exhibit partial order: positional correlations decay fast owing to spatial disorder, whereas the orientational degrees-offreedom remain correlated over long-range. As in the $2 \mathrm{D}$ droplet ensembles reported here, also in gravitational sedimentation of particles in $2 \mathrm{D}$ and $3 \mathrm{D}$, the velocity variance decreases at high densities $^{2,12,26}$. However, so far this generic decrease has lacked a first-principles theoretical explanation. In Brownian quasi-2D suspensions of particles in equilibrium, the dipoles are randomly oriented and there is no symmetry-breaking direction. Hence, at large distances velocity correlations are determined only by two-body interactions ${ }^{11}$. Future studies may reveal how the velocity correlations are coupled to dynamic clustering, which has an important role in setting the fluctuations. We expect our results to have applications in the design of active and self-propelled systems ${ }^{27,28}$ as well as in droplet-based microfluidic devices used in biology and chemistry ${ }^{29,30}$.

\section{Methods}

The channel was made of polydimethylsiloxane elastomer casted on a mould, which was prepared by lithography. After curing at $80^{\circ} \mathrm{C}$ for $1 \mathrm{~h}$, the channel was detached from the mould and irreversibly attached on a polydimethylsiloxane-coated glass slide ${ }^{16,20}$. The carrier fluid was light mineral oil (Sigma, M5904, viscosity $\eta_{\text {oil }}=30 \mathrm{mPa}$, density $\rho_{0}=0.84 \mathrm{~g} \mathrm{ml}^{-1}$ ) with $2 \%(\mathrm{w} / \mathrm{w})$ Span-80 surfactant (Sigma). The dispersed fluid was distilled water. The experiment was imaged by a PCO.Sensicam (PCO) camera for about $100 \mathrm{~s}$ at 21 frames s$^{-1}$. We used a precise tracking algorithm (the Moses-Abadi algorithm) ${ }^{8}$ implemented in Matlab (Mathworks) to analyse the images acquired in the experiment. Each droplet's centre position was tracked and followed between subsequent images to construct its trajectory. The droplet velocities were computed by five-point time derivatives of their $x$ and $y$ positions. To compute the velocity correlations $C_{x}(\mathbf{r})$ we computed the average product of the velocity fluctuations $\delta u_{x}$ of all droplet couples within the channel's central half that were separated by $\mathbf{r}=(r, \theta)$. The velocity fluctuations were defined as the difference between the droplets' individual velocities and the locally measured mean velocity. Owing to the spatial and temporal fluctuations of the mean droplet velocity, it was measured separately for each droplet pair. The mean velocity was defined as the average velocity of droplets within a rectangle of length $W$ along $x$ (centred at the mean $x$ position of the droplet pair) and width $W / 2$ along $y$ (centred at the middle of the channel).

Received 27 June 2013; accepted 20 November 2013; published online 12 January 2014

\section{References}

1. Segrè, P. N., Herbolzheimer, E. \& Chaikin, P. M. Long-range correlations in sedimentation. Phys. Rev. Lett. 79, 2574-2577 (1997).

2. Segrè, P. N., Liu, F., Umbanhowar, P. \& Weitz, D. A. An effective gravitational temperature for sedimentation. Nature 409, 594-597 (2001).

3. Mucha, P. J., Tee, S-Y., Weitz, D. A., Shraiman, B. I. \& Brenner, M. P. A model for velocity fluctuations in sedimentation. J. Fluid Mech. 501, 71-104 (2004)

4. Morfill, G. E. \& Ivlev, A. V. Complex plasmas: An interdisciplinary research field. Rev. Mod. Phys. 81, 1353-1404 (2009).
5. Toner, J., Tu, Y. \& Ramaswamy, S. Hydrodynamics and phases of flocks. Ann. Phys. 318, 170-244 (2005).

6. Zhang, H. \& Be'er, A. Collective motion and density fluctuations in bacterial colonies. Proc. Natl Acad. Sci. USA 107, 13626-13630 (2010).

7. Cavagna, A. et al. Scale-free correlations in starling flocks. Proc. Natl Acad. Sci. USA 107, 11865-11870 (2010).

8. Beatus, T., Tlusty, T. \& Bar-Ziv, R. Phonons in a one-dimensional microfluidic crystal. Nature Phys. 2, 743-748 (2006).

9. Campa, A., Dauxois, T. \& Ruffo, S. Statistical mechanics and dynamics of solvable models with long-range interactions. Phys. Rep. 480, 57-159 (2009).

10. Bouchet, F. \& Barré, J. Statistical mechanics of systems with long range interactions. J. Phys. Conf. Ser. 31, 18-26 (2006).

11. Cui, B., Diamant, H., Lin, B. \& Rice, S. Anomalous hydrodynamic interaction in a quasi-two-dimensional suspension. Phys. Rev. Lett. 92, 258301 (2004).

12. Rouyer, F., Lhuillier, D., Martin, J. \& Salin, D. Structure, density, and velocity fluctuations in quasi-two-dimensional non-Brownian suspensions of spheres. Phys. Fluids 12, 958-963 (2000).

13. Long, D., Stone, H. A. \& Ajdari, A. Electroosmotic flows created by surface defects in capillary electrophoresis. J. Colloid Interface Sci. 212, 338-349 (1999).

14. Suzuki, Y. Y. \& Izuyama, T. Diffusion of molecules in biomembranes. J. Phys. Soc. Jpn 58, 1104-1119 (1989).

15. Thorsen, T., Roberts, R. W., Arnold, F. H. \& Quake, S. R. Dynamic pattern formation in a vesicle-generating microfluidic device. Phys. Rev. Lett. 86, 4163-4166 (2001)

16. Beatus, T., Bar-Ziv, R. H. \& Tlusty, T. The physics of 2D microfluidic droplet ensembles. Phys. Rep. 516, 103-145 (2012).

17. Landau, L. D., Lifshitz, E. M. \& Pitaevskii, L. P. Electrodynamics of continuous media. Course Theor. Phys. 8, 45-47 (1984).

18. Beatus, T., Bar-Ziv, R. \& Tlusty, T. Anomalous microfluidic phonons induced by the interplay of hydrodynamic screening and incompressibility. Phys. Rev. Lett. 99, 124502 (2007).

19. Baron, M., Bławzdziewicz, J. \& Wajnryb, E. Hydrodynamic crystals: Collective dynamics of regular arrays of spherical particles in a parallel-wall channel. Phys. Rev. Lett. 100, 174502 (2008).

20. Beatus, T., Tlusty, T. \& Bar-Ziv, R. Burgers shock waves and sound in a 2D microfluidic droplets ensemble. Phys. Rev. Lett. 103, 114502 (2009).

21. Champagne, N., Vasseur, R., Montourcy, A. \& Bartolo, D. Traffic jams and intermittent flows in microfluidic networks. Phys. Rev. Lett. 105, 044502 (2010)

22. Champagne, N., Lauga, E. \& Bartolo, D. Stability and non-linear response of $1 \mathrm{D}$ microfluidic-particle streams. Soft Matter 7, 11082-11085 (2011).

23. Uspal, W. E. \& Doyle, P. S. Scattering and nonlinear bound states of hydrodynamically coupled particles in a narrow channel. Phys. Rev. E 85, 016325 (2012).

24. Desreumaux, N., Caussin, J-B., Jeanneret, R., Lauga, E. \& Bartolo, D. Hydrodynamic fluctuations in confined particle-laden fluids. Phys. Rev. Lett. 111, 118301 (2013).

25. Uspal, W. E., Burak Eral, H. \& Doyle, P. S. Engineering particle trajectories in microfluidic flows using particle shape. Nature Commun. 4, 2666 (2013).

26. Snabre, P., Pouligny, B., Metayer, C. \& Nadal, F. Size segregation and particle velocity fluctuations in settling concentrated suspensions. Rheol. Acta $\mathbf{4 8}$, 855-870 (2009).

27. Brotto, T., Caussin, J-B., Lauga, E. \& Bartolo, D. Hydrodynamics of confined active fluids. Phys. Rev. Lett. 110, 038101 (2013).

28. Palacci, J., Sacanna, S., Steinberg, A. P., Pine, D. J. \& Chaikin, P. M. Living crystals of light-activated colloidal surfers. Science 339, 936-40 (2013).

29. Theberge, A. B. et al. Microdroplets in microfluidics: An evolving platform for discoveries in chemistry and biology. Angew. Chem. Int. Ed. Engl. 49, 5846-68 (2010).

30. Joensson, H. N. \& Andersson Svahn, H. Droplet microfluidics-a tool for single-cell analysis. Angew. Chem. Int. Ed. Engl. 51, 12176-92 (2012).

\section{Acknowledgements}

This work was supported by a Yeda-Sela Grant (R.H.B-Z). T.B. was supported by the Cross Disciplinary Postdoctoral Fellowship of the Human Frontier Science Program. T.T. is the Helen and Martin Chooljian Founders Circle Member in the Simons Center for Systems Biology of the Institute for Advanced Study, Princeton.

\section{Author contributions}

All authors contributed to all aspects of this work.

\section{Additional information}

Supplementary information is available in the online version of the paper. Reprints and permissions information is available online at www.nature.com/reprints. Correspondence and requests for materials should be addressed to R.H.B-Z. or T.T.

\section{Competing financial interests}

The authors declare no competing financial interests. 